\title{
Tsafon
}

Revue d'études juives du Nord

$80 \mid 2020$

Varia

\section{« Les Nations du monde narguent (les enfants d')Israël en leur disant... »}

Étude d'un motif aggadique dans son contexte historique

José Costa

\section{OpenEdition}

\section{Journals}

Édition électronique

URL : https://journals.openedition.org/tsafon/3096

DOI : 10.4000/tsafon.3096

ISSN : 2609-6420

\section{Éditeur}

Association Jean-Marie Delmaire

Édition imprimée

Date de publication : 1 décembre 2020

Pagination : 13-35

ISSN : 1149-6630

\section{Référence électronique}

José Costa, « « Les Nations du monde narguent (les enfants d') Israël en leur disant... » », Tsafon [En ligne], 80 | 2020, mis en ligne le 01 décembre 2020, consulté le 24 juin 2021. URL : http:// journals.openedition.org/tsafon/3096 ; DOI : https://doi.org/10.4000/tsafon.3096 


\section{« Les Nations du monde narguent (les enfants d')Israël en leur disant... »}

\section{Étude d'un motif aggadique dans son contexte historique}

José Costa*

On distingue habituellement deux formes d'antijudaïsme dans l'Antiquité, celui des païens (souvent appelé «judéophobie ») ${ }^{1}$ et celui des chrétiens (que désigne le terme «antijudaïsme», employé au sens étroit). Les relations entre ces deux antijudaïsmes ont fait l'objet d'appréciations variées ${ }^{2}$. Sur certains motifs, la continuité est évidente : par exemple, le contraste entre la prétention qu'ont les juifs à être un peuple élu et les humiliations diverses qu'ils subissent sur la scène de l'histoire ${ }^{3}$. Pour l'essentiel cependant, les deux discours ne mettent pas l'accent sur les mêmes thèmes. L'antijudaïsme grec insiste surtout sur l'insociabilité (amixia) des juifs (ou leur misanthropie) et sur leur

\footnotetext{
*Université de Paris 3.

1 Voir P. Schäfer, Judeophobia. Attitudes toward the Jews in the Ancient World, Cambridge-Londres, Harvard University Press, 1997.

${ }^{2}$ Voir J. Gager, The Origins of Antisemitism : Attitudes toward Judaism in Pagan and Christian Antiquity, Oxford, Oxford University Press, 1983 ; S. C. Mimouni, « Pour une histoire de la séparation entre les communautés chrétiennes et les communautés pharisiennes (ca. 70-135 de notre ère) » (version actualisée, consultée sur le site academia, https://www.academia.edu/40159110), p. 4 et n. 8; G. Stroumsa, «De l'antijudaïsme à l'antisémitisme dans le christianisme ancien », dans Le rire du Christ. Essais sur le christianisme antique, Paris, Bayard, 2006, p. 139-182 et plus particulièrement p. 144-149 ; J. Mélèze Modrzejewski, Un peuple de philosophes. Aux origines de la condition juive, Paris, Fayard, 2011, p. 348.

${ }^{3}$ Voir R. Kimelman, «Rabbi Yohanan and Origen on the Song of Songs : The ThirdCentury Jewish-Christian Disputation », The Harvard Theological Review 73, 1980, p. 588 et n. 93-94.
} 
athéisme (atheotês). À ces deux critiques, qu'ils reprennent, les Romains ont ajouté le reproche de comploter contre la sûreté de l'État ${ }^{4}$. Pour les Pères de l'Église, les juifs, qui sont aveugles au vrai sens de l'Écriture, n'ont pas reconnu la messianité de Jésus, sont coupables de déicide et ont été punis dans l'histoire pour leurs fautes ${ }^{5}$.

Dans les travaux sur l'antijudaïsme païen et chrétien, la littérature rabbinique n'est guère sollicitée. Pour prendre un exemple emblématique, l'ouvrage de P. Schäfer Judeophobia ne cite aucun texte en provenance des rabbins. Ces derniers évoquent pourtant à plusieurs reprises l'hostilité des Nations à l'égard d'Israël. S. Stern a réuni en quelques pages les extraits qui lui ont semblé les plus significatifs ${ }^{6}$. C'est une halakha qu'Esaü hait Jacob ${ }^{7}$. Le nom de la fête romaine des Saturnalia comme le terme senator se réfèrent l'un et l'autre à la haine des Romains à l'égard des juifs ${ }^{8}$. Celle-ci s'exprime aussi tous les soixante-dix ans dans une cérémonie où un homme en bonne santé chevauche un homme malade ${ }^{9}$. Les Romains tournent aussi en dérision le shabbat et la septième année dans les théâtres ${ }^{10}$. Ils se moquent d'Israël chaque jour $^{11}$. En fait, les Nations attaquent Israël à cause de la Tora qu'il pratique : si Israël était incirconcis et idolâtre, il ne rencontrerait aucune hostilité de leur part ${ }^{12}$. Elles attaquent Israël à défaut de pouvoir attaquer

\footnotetext{
${ }^{4}$ Voir J. Mélèze Modrzejewski, Un peuple de philosophes..., op. cit., p. 36-38, 90-92, 345-348. G. Stroumsa, qui s'appuie sur un article de L. H. Feldman, propose la liste suivante : " haine de l'humanité, crédulité, double allégeance, prosélytisme agressif... et mendicité » (G. Stroumsa, « De l'antijudaïsme à l'antisémitisme... », op. cit., p. 144145). La pratique de la circoncision suscitait également une vive répulsion chez les Grecs et les Romains et elle a fait l'objet de débats nourris chez les chrétiens (ainsi que chez les [autres] juifs) : voir sur ce point S. C. Mimouni, La circoncision dans le monde judéen aux époques grecque et romaine. Histoire d'un conflit interne au judaïsme, Paris-Louvain, Peeters, 2007.

${ }^{5}$ Voir G. Stroumsa (qui s'appuie sur une étude de G. I. Langmuir), « De l'antijudaïsme à l'antisémitisme... ", op. cit., p. 148-149. Dans un article qui porte sur la période entre 70 et 135, S. C. Mimouni distingue les thèmes suivants : le conflit sur les observances (autour de la loi), celui sur la messianité et celui sur le rôle d'Israël dans le «temps du salut » : " Pour une histoire de la séparation... », op. cit., p. 16-19.

${ }^{6}$ S. Stern, Jewish Identity in Early Rabbinic Writings, Leyde-New York-Cologne, Brill, 1994, p. 47-50.

${ }^{7}$ Sifre Ba-midbar, 69.

${ }_{\mathrm{j}}^{\mathrm{j}}$ 'Aboda Zara, 1, 2 ; Be-reshit Rabba, 67, 8 .

${ }^{9} \mathrm{~b}$ 'Aboda Zara, 11b. Pour d'autres textes sur la haine des Romains et des Nations à l'égard d'Israël, voir également S. Stern, Jewish Identity..., op. cit., p. 48, n. 358.

10 Ekha Rabba, petihta, 17 et 3, 5.

11 Ba-midbar Rabba, 11, 1.

12 Midrash Tanhuma, Mațtot, 3.
} 
Dieu $^{13}$. Elles l'asservissent et consacrent leur temps à réfléchir au mal qu'elles peuvent encore lui faire ${ }^{14}$. D'autres textes affirment que les Nations éprouvent à l'égard d'Israël admiration, jalousie et peur ${ }^{15}$. Peu d'entre eux renvoient clairement aux prétentions chrétiennes à l'égard d'Israël, sauf un passage où les Nations déclarent être le vrai Israël ${ }^{16}$.

Il est frappant de voir que les textes cités par Stern ne recoupent qu'imparfaitement les thèmes de l'antijudaïsme païen et chrétien que nous avons mentionnés plus haut. D'autres passages sont peut-être plus parlants, comme celui qui met en scène Rabbi 'Aqiba à Rome dans le domicile d'un dignitaire romain ${ }^{17}$. Alors que son hôte lui envoie deux belles femmes pour passer la nuit avec lui, il ne les regarde pas et va même, selon certaines versions, jusqu'à émettre un crachat $^{18}$. Le dignitaire romain lui dit alors : " Pourquoi n'as-tu pas agi à l'égard de ces femmes comme les hommes agissent habituellement avec les belles femmes ? Ne sont-elles pas humaines comme toi ? Celui qui t'a créé ne les a-t-elle pas créées ? ${ }^{19}$. Il reprend un discours universaliste, promu notamment par les stoïciens et reproche à Rabbi 'Aqiba sa misanthropie profonde, ce qui rejoint l'un des griefs majeurs que les Grecs et les Romains avaient à l'égard des juifs. Devant le plaidoyer universaliste du Romain, Rabbi 'Aqiba assume pleinement son différentialisme en évoquant une répulsion d'ordre physique : «Que pouvais-je faire ? Leur odeur est venue sur moi à partir de la viande de carcasses et de bêtes déchirées et de viande de porc $»^{20}$. Le corpus de douze textes, que nous allons maintenant commenter, mérite également d'être considéré de près. Il n'a jamais fait l'objet jusqu'à présent d'une étude exhaustive ${ }^{21}$.

\footnotetext{
13 Midrash Tanhuma, Pequde, 4.

14 Midrash Tanhuma, Toledot, 5.

${ }^{15}$ Voir S. Stern, Jewish Identity..., op. cit., p. 47, 50.

${ }^{16}$ Shir ha-shirim Rabba, 7, 3, 3. La polémique implicite à l'égard de l'exégèse chrétienne occupe une place beaucoup plus importante dans la littérature rabbinique : voir, par exemple, l'ouvrage récent de E. Grypeou, H. Spurling, The Book of Genesis in Late Antiquity. Encounters between Jewish and Christian Exegesis, Leyde-Boston, Brill, 2013.

${ }^{17}$ Abot de-rabbi Natan, A, 16.

${ }^{18}$ Voir Avot de-Rabbi Natan. Synoptische Edition beider Versionen, H. J. Becker (éd.), Tübingen, Mohr-Siebeck, 2006 p. 164-167.

${ }^{19}$ Ms Oxford Heb. c. 24 (Avot de-Rabbi Natan..., op. cit., p. 166).

${ }^{20}$ Ms Oxford Heb. c. 24 (Avot de-Rabbi Natan..., op. cit., p. 166). Sur l'ensemble du passage, voir J. W. Schofer, The Making of a Sage. A Study in Rabbinic Ethics, Madison-Londres, The University of Wisconsin Press, 2005, p. 110, 240.

${ }^{21}$ Certains des textes ont déjà été commentés par I. Baer, E. Urbach, R. Kimelman et M.

J. Kister, comme nous le verrons dans la suite du développement.
} 
1. j Berakhot 2, 2, ms Leyde, Scaliger $3^{22}$

Quand (Rabbi Yohanan) allait au bain, dès qu'il arrivait auprès du responsable du vestiaire, il les ${ }^{23}$ enlevait. Rabbi Yiṣhaq a dit: Il les revêtait jusqu'à (ce qu'il arrive) devant Jacob le gardien du bain. Quand il sortait du bain, on (les) lui donnait. Quand on les lui apportait, il disait cette parole : Deux coffres se déplaçaient dans le désert avec Israël, le coffre du Vivant des mondes et le coffre de Joseph ${ }^{24}$. Les Nations disaient: Que sont ces deux coffres? (Les enfants d')Israël ${ }^{25}$ répondaient: C'est le coffre de Joseph avec le coffre du Vivant des mondes. Les Nations du monde narguaient (les enfants d')Israël en leur disant : Est-il possible au coffre du mort de se déplacer avec le coffre du Vivant des mondes? (Les enfants d')Israël répondaient : C'est parce que celui-ci ${ }^{26}$ a observé ce qui est écrit dans celui-là ${ }^{27}$. Pourquoi disait-il cette parole $^{28}$ ? Rabbi Hanina a dit : C'est parce qu'il (voulait) dire une parole de Tora. Rabbi Mana lui objecta: Est-ce qu'il n'avait pas une autre parole de Tora à dire que celle-là ? En fait, c'étaient des remontrances qui (veulent) dire : Joseph n'a mérité la royauté que parce qu'il a observé les commandements du Saint, béni soit-il. Nous n'avons mérité toute cette gloire que parce que nous avons observé les commandements du Saint, béni soit-il. Or, vous voulez que nous annulions un commandement ${ }^{29}$ !

2. Be-reshit Rabba, 1, 2, ms Vatican, Biblioteca Apostolica ebr., 60 Rabbi Yehoshua' de Sikhnin a dit l'ouverture (suivante) au nom de Rabbi Lévi : «Il a raconté la force de ses actions à son peuple, pour leur donner l'héritage des Nations » (Ps 111, 6). Pour quelle raison le Saint,

\footnotetext{
${ }^{22}$ On trouve une version plus ancienne de l'histoire des deux coffres dans T Sota, 4, 3 et Mekhilta de-rabbi Yishma'el, Be-shallah, Wa-yehi, Petihta, qui ne met pas en scène des Nations qui narguent Israël, mais de simples passants ('oberin we-shabin) qui s'adressent à lui. Les passants posent cependant deux questions semblables à celles des Nations dans notre texte. Les traductions sont de l'auteur.

${ }^{23}$ Ses tefillin.

${ }^{24}$ Coffre traduit l'hébreu aron. Dans un cas, le terme désigne l'arche d'alliance et dans l'autre, le cercueil de Joseph.

${ }^{25}$ Le texte hébreu emploie le nom Israël suivi par un verbe au pluriel, ce qui n'est pas facile à rendre en français, d'où notre traduction : «(les enfants d')Israël ». Nous avons procédé de même dans la traduction des autres textes.

${ }^{26}$ Joseph.

${ }^{27}$ Le coffre, c'est-à-dire l'arche qui contient la Tora.

${ }^{28}$ Pourquoi Rabbi Yohanan disait-il cet enseignement sur les deux coffres au moment où il récupérait ses tefillin?

${ }^{29}$ Rabbi Yohanan critique ici les juifs qui négligent le commandement des tefillin.
} 
béni soit-il, a-t-il révélé à Israël ce qui a été créé le premier jour et ce qui a été créé le deuxième jour ? C'est à cause des Nations du monde ${ }^{30}$, afin qu'elles ne narguent pas (les enfants d')Israël en leur disant : N'êtes-vous pas une nation de voleurs ? C'est surprenant ${ }^{31}$ ! (Les enfants d')Israël (pourraient) leur répondre ${ }^{32}$ en disant: Et vous, n'avez-vous pas une chose volée dans vos mains ? «(De même, les Avvéens qui habitaient dans les villages jusqu'à Gaza), les Kaftorîm qui sont sortis de Kaftor ne les ont-ils pas exterminés et n'ont-ils pas habité à leur place ? »(Dt 2, 23). Le monde et ce qu'il contient sont (la propriété) du Saint, béni soitil. Quand il l'a voulu, il vous l'a donnée et quand il l'a voulu, il l'a prise de vous ${ }^{33}$ et nous l'a donnée. C'est ce qui est écrit (dans Ps 111,6) : « pour leur donner l'héritage des Nations ». "Il a raconté la force de ses actions », il leur a raconté le commencement ${ }^{34}$, ainsi qu'il est dit : « $\mathrm{Au}$ commencement, Dieu créa le ciel et la terre $»(\mathrm{Gn} 1,1)$.

3. Be-reshit Rabba, 79, 7, ms Vatican, Biblioteca Apostolica ebr. 30 (troisième main)

«Et il a acquis la part du champ...» $(\mathrm{Gn} 33,19)^{35}$. Rabbi Yuda berabbi Simon a dit: C'est l'un des trois lieux sur lesquels les Nations du monde ne peuvent pas narguer (les enfants d')Israël en leur disant : Ce sont des choses volées dans votre main. Les voici: la caverne de Makpêla, le Temple et la tombe de Joseph. La caverne de Makpêla, « Et Abraham entendit Efrôn (et Abraham pesa à Efrôn l'argent dont il avait parlé...)» (Gn 23, 16). Le Temple, «Et David donna à Ornan pour le lieu des sicles (d'or, d'un poids de six cents) » $(1 \mathrm{Ch} 21,25)$. La tombe de Joseph, «Et il a acquis la part du champ... »" ${ }^{36}(\mathrm{Gn} 33,19)$.

\footnotetext{
${ }^{30}$ Dans Be-reshit Rabba, 61, 7, ce sont les Cananéens eux-mêmes qui réclament, devant Alexandre le Grand, que les juifs leur rendent leur terre. Voir également Talmud Babli, Sanhedrin, 91a. C'est un Sage, du nom de Gebiha, qui leur répond.

${ }^{31}$ Une telle affirmation aurait été surprenante, tant elle n'est pas fondée.

32 Nous comprenons: Israël pourrait répondre aux Nations, si ces dernières le narguaient quand même. Lit. : «(Les enfants d')Israël leur répondent en disant ».

${ }^{33}$ La terre de Canaan.

${ }^{34}$ Ps 111, 6 signifie donc : « Il (= Dieu) a raconté la force de ses actions (= dans le récit de la création) pour leur donner (= à Israël) l'héritage des Nations (= la terre de Canaan) ». Le récit de la création permet de légitimer la conquête de Canaan par les Hébreux.

${ }^{35}$ Jacob achète aux fils de Hamor une parcelle de terrain qui se trouve en face de la ville de Sichem.

${ }^{36}$ Le champ acquis par Jacob en face de la ville de Sichem est aussi le lieu où les ossements de Joseph ont été enterrés (Jos 24, 32).
} 
4. Be-reshit Rabba, 88, 1, ms Vatican, Biblioteca Apostolica ebr. 30 (première main)

«Et ce fut après ces choses que péchèrent (l'échanson du roi d'Égypte et le panetier envers leur maître, le roi d'Égypte)» (Gn 40, 1). «De tous mes péchés, sauve-moi, (ne me place pas comme une chose honteuse devant l'insensé [herpat nabal]) » (Ps 39, 9). Rabbi Hama beRabbi Hanina et Rabbi Shemu'el bar Naḥman (interprètent différemment ce verset) ${ }^{37}$. Rabbi Hama be-Rabbi Hanina a dit : Les Nations du monde n'auraient pas dû avoir en leur sein des gens abattus et repoussés. Pourquoi y a-t-il (alors) en leur sein des gens abattus et repoussés ${ }^{38}$ ? C'est afin qu'elles ne narguent pas (les enfants d')Israël en leur disant : N'êtes-vous pas une nation de gens abattus et repoussés? C'est surprenant $^{39}$ ! C'est à cause du verset « ne me place pas comme une chose honteuse ${ }^{40}$ devant l'insensé ${ }^{41}$ (Ps 39, 9) ${ }^{42}$. Rabbi Shemu'el bar Naḥman a dit : Les Nations du monde n'auraient pas dû avoir en leur sein des gens affectés par la gale. Pourquoi y a-t-il (alors) en leur sein des gens affectés par la gale ? C'est afin qu'elles ne narguent pas (les enfants d')Israël en leur disant : N'êtes-vous pas une nation de lépreux ? C'est à cause du verset « ne me place pas comme une chose honteuse ${ }^{43}$ (devant l'insensé) » (Ps 39, 9).

5. Be-reshit Rabba, 95, 6, ms Vatican, Biblioteca Apostolica ebr. 30 (première main)

«Et quant au peuple, il le fit passer vers (d'autres) villes (d'une extrémité du territoire de l'Égypte à son [autre] extrémité) »(Gn 47,

\footnotetext{
${ }^{37}$ Ils appliquent cependant tous les deux le verset à Israël. Ce dernier a péché (ou va pécher) et subit (ou subira) des souffrances expiatoires, qui peuvent faire de lui un objet de honte devant les Nations. Israël demande donc à Dieu de lui éviter cette honte, ce que Dieu ne peut faire qu'en envoyant des souffrances similaires sur les Nations. Les deux lectures de Ps 39, 9 par Rabbi Ḥama be-Rabbi Hanina et Rabbi Shemu'el bar Naḥman sont suivies par une troisième lecture du même verset, anonyme, qui l'applique à Joseph. C'est cette troisième lecture qui a un rapport avec le verset de départ (Gn 40,1).

${ }^{38}$ Le monde présent est en effet la part des Nations, qui n'auraient donc pas dû subir ce genre de désagrément. C'est uniquement dans le monde futur que les Nations doivent être punies pour leurs fautes.

${ }^{39}$ Il faut probablement comprendre : ce serait surprenant que les Nations s'expriment ainsi, car elles ont, elles aussi, leurs gens abattus et repoussés.

${ }^{40}$ Pour Rabbi Hama be-Rabbi Hanina, la chose honteuse est d'être abattu et repoussé.

${ }^{41}$ Les insensés sont les gens des Nations.

${ }^{42} \mathrm{Si}$ contre toute attente les Nations sont frappées aussi, c'est à cause de la demande qu'Israël a adressée à Dieu, dont témoigne Ps 39, 9.

${ }^{43}$ Pour Rabbi Shemu'el bar Nahman, la chose honteuse est d'avoir la gale ou la lèpre.
} 
$21)^{44}$. Rabbi Shim'on ben Yohay enseigne : Que dois-je interpréter et dire (à partir du verset) «Et quant au peuple, il le fit passer vers (d'autres) villes » (Gn 47, 21) ? En fait, (Joseph a agi ainsi), afin qu'ils ne narguent pas (les enfants d')Israël en leur disant : N'êtes-vous pas une nation de gens exilés et déplacés ? C'est pourquoi il prenait (des gens) d'ici et les mettait là-bas (et) il prenait (des gens) de là-bas et les mettait ici.

6. Pesiqta de rab Kahana, 10, 6, ms Oxford, Bodleian Library $151: 1^{45}$

«C'est un jardin clos que ma sœur, (ma) fiancée une source fermée, une fontaine scellée » $(\mathrm{Ct} 4,12)$. Rabbi Yehuda bar Simon (a dit) au nom de Rabbi Yehoshua' ben Lévi : (Parabole) d'un roi qui avait des filles majeures ${ }^{46}$ et il ne parvenait pas à les marier. Il partit pour la province de la mer. Ses filles se dressèrent, se prirent elles-mêmes en charge et épousèrent des hommes. Chacune d'entre elles prit le sceau de son mari et son cachet. Après (quelques) jours, le roi revint de la province de la mer et entendit la rumeur des gens qui calomniaient ses filles et disaient que les filles du roi s'étaient conduites comme des prostituées. Qu'a-t-il fait? Il fit une proclamation qui disait: Que tout le peuple (se rassemble) dans le champ (qampon)! Il appela son premier gendre $^{47}$. Il lui dit : Qui es-tu? Il lui répondit : Je suis ton gendre. Il sortit son sceau. Il lui demanda: De qui est ce (sceau)? Il lui répondit : C'est le mien. Il sortit son cachet. Il lui demanda : De qui est ce (cachet) ? Il lui répondit: C'est le mien. De même pour le second (gendre) et de même pour le troisième (gendre). Le roi a dit: Mes filles se sont prises ellesmêmes en charge et se sont mariées, alors que vous dites que les filles du roi se sont conduites comme des prostituées ${ }^{48}$. De même, (le verset de $\mathrm{Ct}$ 4, 12 a été écrit) ${ }^{49}$, parce que les Nations du monde narguaient (les

\footnotetext{
${ }^{44}$ Le verset décrit l'action de Joseph au moment de la famine. Il est problématique : pourquoi Joseph déplace-t-il des Égyptiens d'une ville à l'autre?

${ }^{45}$ Parallèle dans Shir ha-shirim Rabba, 4, 12, 1.

${ }^{46}$ Shir ha-shirim Rabba: une fille majeure et une fille mineure.

${ }^{47}$ Shir ha-shirim Rabba: le roi commence par accuser ses filles de s'être mal conduites. Elles montrent alors le sceau et le cachet de leurs maris. Le roi interroge ensuite les maris, comme c'est le cas ici.

${ }^{48}$ Shir ha-shirim Rabba: le roi ajoute qu'il va châtier les calomniateurs.

${ }^{49}$ Le verset de $\mathrm{Ct}$ 4, 12 est compris comme une proclamation de Dieu (l'amant) sur la pureté sexuelle d'Israël (la femme aimée), alors qu'il était en Égypte : comme le dit explicitement la suite du texte de la Pesiqta de-rab Kahana (non traduite ici), le « jardin clos » se réfère aux vierges, la "source fermée » aux femmes mariées et la "fontaine
} 
enfants d')Israël en leur disant qu'ils étaient les fils des Égyptiens : Ils dominaient (toutes) les vies ${ }^{50}$ d'Israël, combien plus leurs femmes ${ }^{51}$ ! Rabbi Hosha'ya a dit : À ce moment, le Saint, béni soit-il, appela l'ange qui est chargé de la fécondation. Il lui dit: Va et dessine-moi la forme des embryons à la ressemblance de leurs pères, c'est ce qui est écrit : «Pour Ruben (...) familles issues de Ruben (ha-re'ubeni)» (Nb 26, 5 et 7), «Pour Siméon (...) familles issues de Siméon (ha-shim 'oni) » (Nb 26, 12 et 14) ${ }^{52}$. Rabbi Merinos be-rabbi Hosha'ya a dit: (C'est ce que) disent les gens : Mon cher fils, mon cher visage, mon apparence ${ }^{53}$. Rabbi Idi a dit: Il y a un he au début du mot et un yod à la fin ${ }^{54}$. Yah témoigne sur eux qu'ils sont les fils de leurs pères. Quelle en est la preuve scripturaire? «Car là-bas sont montées les tribus, les tribus de Yah, témoignage pour Israël » (Ps 122, 4).

\section{$151: 1^{55}$}

7. Pesiqta de rab Kahana, 19, 4, ms Oxford, Bodleian Library

«C'est moi, c'est moi qui vous console » (Is 51, 12). Rabbi Abba bar Kahana (a dit) au nom de Rabbi Yoḥanan : (Parabole) d'un roi qui a épousé une dame de qualité et lui a écrit un contrat de mariage avantageux : Je ferai pour toi tel et tel dais, je te donnerai tel et tel bijou, je te donnerai tel et tel trésor ${ }^{56}$. Il la laissa et il partit pour la province de la mer. Il demeura là-bas de nombreuses années. Ses compagnes la narguaient et lui disaient: Jusqu'à quand vas-tu rester assise (à l'attendre) ? Prends pour toi un (autre) mari, tant que tu es jeune et que tu as de la force en to ${ }^{57}$. Elle entrait (alors) dans sa maison, prenait son contrat de mariage et le lisait, ce qui la consolait. Après de (nombreux) jours, le roi revint de la province de la mer. Il lui dit: Ma fille, je suis

scellée » aux hommes. Dans Shir ha-shirim Rabba, le verset est placé explicitement dans la bouche de Dieu.

${ }^{50}$ Shir ha-shirim Rabba : leurs actions.

${ }^{51}$ Shir ha-shirim Rabba: leurs corps et leurs femmes. Cette version cite aussi Ex 1, 13.

52 Pourquoi insister sur le fait que ces familles sont «rubénites» (re'ubeni) ou « siméonites » (shim 'oni), alors que le nom de l'ancêtre éponyme a déjà été mentionné ? C'est pour nous montrer que tous les chefs des familles issues de Ruben ou Siméon avaient la même apparence physique que leur ancêtre éponyme (voir Shir ha-shirim Rabba).

${ }^{53} \mathrm{Ce}$ proverbe insiste aussi sur la ressemblance physique entre le fils et son père (ou son ancêtre) et les trois mots employés se terminent par un yod comme re'ubeni et shim 'oni.

${ }^{54} \boldsymbol{H}$ a-re'ubeni, ha-shim 'oni. I(Y) + $\mathrm{H}=$ le nom divin Yah.

${ }^{55}$ Parallèle dans Ekha Rabba, 3, 7.

${ }^{56}$ Ekha Rabba ne cite que deux biens précieux : le dais et la pourpre.

${ }^{57}$ Ekha Rabba: le roi t'a abandonné et ne reviendra pas vers toi. 
étonné que tu m'aies attendu toutes ces années. Elle lui répondit : Mon seigneur, le roi, s'il n'y avait pas eu le contrat de mariage avantageux que tu as écrit pour moi, mes compagnes m'auraient déjà séparée de toi. De même, (le verset de Lv 26, 9 a été écrit), parce que, dans le monde présent, les Nations du monde ${ }^{58}$ narguent (les enfants d')Israël en leur disant : Jusqu'à quand serez-vous opprimés pour votre Dieu, donnerezvous votre vie pour lui et serez-vous tués pour lui ? Combien de peine at-il fait venir sur vous? Combien de mépris a-t-il fait venir sur vous? Combien de souffrances a-t-il fait venir sur vous? Venez auprès de nous et nous ferons de vous des ducs, des gouverneurs et des commandants en chef $^{59}$. (Les enfants d')Israël ${ }^{60}$ entrent (alors) dans les synagogues ${ }^{61}$ et dans les maisons d'étude. Ils prennent le rouleau de la Tora et lisent en lui (le verset suivant): «Et je me déplacerai au milieu de vous, je vous ferai fructifier, je vous multiplierai et j'établirai mon alliance avec vous » (Lv 26, 9). Ils sont (ainsi) consolés ${ }^{62}$.

\section{Wa-yiqra Rabba, 27, 8, ms Londres, British Library $340^{63}$}

«Par (l'objet de) leur mauvaise (conduite) (be-ra'atam), ils réjouiront le roi » $(\mathrm{Os} 7,3)^{64}$. Qu'a (de particulier) le bœuf pour avoir été

\footnotetext{
${ }^{58}$ Ekha Rabba les identifie probablement aux Babyloniens.

${ }^{59}$ Ce passage rappelle $\mathrm{Jb} 2,9-10$, où la femme de Job incite son mari, frappé par de multiples souffrances, à renier Dieu. Dans Ekha Rabba, le discours des Nations est différent : il insiste sur le fait que Dieu a voilé sa face devant Israël, qu'il a fait monter sa Shekhina loin d'eux et qu'il ne reviendra pas. Un texte de Shir ha-shirim Rabba $(7,1,2)$ présente en revanche la même structure que notre texte: 1 . Une série de questions autour du thème «jusqu'à quand souffrirez-vous pour Dieu?»; 2. L'incitation à rejoindre les Nations et à obtenir des fonctions élevées auprès d'elles.

${ }^{60}$ Dans Ekha Rabba, Israël commence par pleurer et gémir avant d'entrer dans les synagogues...

${ }^{61}$ La synagogue est le lieu de l'intimité : voir, par exemple, Talmud Babli, Ta 'anit, 16a.

${ }^{62}$ Toutes les promesses, énoncées dans Lv 26, 9 (le verset cité mélange Lv 26, 9 et 12), seront réalisées au moment du retour de l'époux divin dans les temps eschatologiques. Leur lecture régulière permet à Israël de tenir bon pendant la longue attente de l'exil. Le verset de départ, Is 51, 12, fait également allusion à cette consolation présente, car il est formulé de manière redondante (avec la double mention du pronom personnel « moi ») : "C'est moi, c'est moi qui vous console ", c'est moi, Dieu, qui vous console dans le monde présent et c'est moi, Dieu, qui vous consolerai dans le monde futur.

${ }^{63}$ Parallèle dans Pesiqta de-rab Kahana, 9.

${ }^{64}$ Le contexte parle des péchés commis par les habitants du royaume du Nord, qui réjouissent leur roi, aussi corrompu qu'eux. Pour le Midrash cependant, le roi n'est autre que Dieu, qui dit juste avant, dans le verset 2, «(leurs mauvaises actions) sont devant ma face ». Il faut donc comprendre ainsi Os 7, 3: ils ont péché par le bouf (la faute du veau d'or) et c'est par ce même bœuf qu'ils m'ont réjoui par la suite (quand il est devenu la première de toutes les offrandes). Comme le texte de Wa-yiqra Rabba affirme l'innocence d'Israël dans l'affaire du veau d'or, dont la responsabilité incombe
} 
fait la première de toutes les offrandes? Rabbi Lévi a dit: (Parabole d'une) dame de qualité, victime d'une mauvaise rumeur (lui prêtant une relation) avec l'un des Grands du royaume. Le roi enquêta sur les paroles (calomniatrices) et les trouva dénuées de toute réalité. Que fit le roi ? Il fit un repas et fit s'asseoir cet homme à la première place des invités. Tout cela pourquoi ? C'est parce que le roi avait enquêté sur les paroles (calomniatrices) et les avait trouvées dénuées de toute réalité. De même, les Nations du monde narguaient (les enfants d')Israël en leur disant : Vous avez fait le veau. Le Saint, béni soit-il, enquêta sur les paroles (calomniatrices) et les trouva dénuées de toute réalité ${ }^{65}$. C'est pourquoi (le bœuf) est devenu la première de toutes les offrandes : « Un bœuf, un mouton ou une chèvre » ( Lv 22, 27). Rab Huna et Rab Idi (ont dit) au nom de Rabbi Shemu'el bar Naḥman: Israël est innocent de cette (mauvaise) action, car si (les enfants d')Israël avaient fait le veau, on aurait dû leur dire : Voici nos dieux Israël. En fait, les prosélytes qui sont montés avec Israël d'Égypte, « et un mélange nombreux (de personnes) (monta) aussi (avec eux) » (Ex 12, 38), ce sont eux qui ont fait le veau et ils les narguaient en leur disant : « Voici tes dieux Israël » $(\operatorname{Ex~32,4})^{66}$.

\section{Wa-yiqra Rabba, 33, 6, ms Londres, British Library $340^{67}$}

Il est écrit : «Moi, garde (shemor) (ce que dit) la bouche du roi, (et sur la parole du serment de Dieu)» (Qo 8, 2). Rabbi Lévi a dit : « Moi » (Qo 8, 2) ${ }^{68}$, je garderai (eshmor) (ce que dit) la bouche du roi des rois des rois, le Saint, béni soit-il, cette parole qu'il a dite au Sinaï : « Je suis l'Éternel ton Dieu » (Ex 20, 2). «Et sur la parole» (Qo 8, 2), ce sont les

à des mauvais prosélytes, on peut préciser ainsi le sens du verset: c'est par le mal apparent qu'ils ont commis, la faute du veau d'or, qu'ils ont réjoui le roi, puisque ce dernier a pu les innocenter et faire par la suite du bœuf, en tant que première offrande, le signe même de leur innocence.

${ }^{65} \mathrm{Ce}$ sont surtout les rabbins qui mènent l'enquête dans la dernière partie du texte.

${ }^{66}$ En disant : «voici tes dieux Israël », les instigateurs du veau d'or ont révélé leur origine non-juive, puisqu'ils s'adressent à Israël comme s'ils n'en faisaient pas partie.

${ }^{67}$ Parallèles dans Talmud Yerushalmi, Sanhedrin, 3, 5 ; Shir ha-shirim Rabba, 2, 14, 1 ; Pesiqta Rabbati, 22; Qohelet Rabba, 8, 2. Aucun ne comporte la conclusion eschatologique de notre texte, introduite par " parce que dans le monde présent... ».

${ }^{68}$ Le commentaire de Rabbi Lévi met l'accent sur le mot «moi » (ani) qui semble ne pas être à sa place dans le verset (on complète généralement le texte de la manière suivante pour le rendre intelligible : «Moi [= c'est Salomon qui parle], [je dis :] Garde [ce que dit] la bouche du roi...). Il fait en fait référence à un autre «moi » (anokhi) dans Ex 20, 2 : «Je (anokhi) suis l'Éternel, ton Dieu... ». Le verset de Qo 8, 2 signifie donc que le roi Salomon gardera la parole d'un roi plus élevé, celui qui a dit: « Moi» au Mont Sinaï. 
dix paroles ${ }^{69}$. «Le serment de Dieu » (Qo 8, 2), « tu n'invoqueras pas le nom de l'Éternel ton Dieu en vain » (Ex 20,7), parce que dans le monde présent $^{70}$, les Nations du monde narguent Israël, mais, dans les temps futurs, je leur donnerai à manger de leur (propre) chair et ils s'enivreront de leur (propre) sang, car il est écrit (en Is 49, 26) : «Je donnerai à manger leur chair à ceux qui te narguent et comme d'un vin nouveau, ils s'enivreront de leur sang ».

\section{Shir ha-shirim Rabba, 1, 6, 3, ms Vatican, Biblioteca} Apostolica ebr. 76

« $\mathrm{Si}$ je suis noiraude, c'est parce que le soleil m'a brunie » $(\mathrm{Ct} 1,6)^{71}$. (...) Histoire d'une femme de la ville qui avait une servante éthiopienne. (La servante) descendit remplir (son seau) à la source, elle et sa compagne ${ }^{72}$. Elle dit à sa compagne : Ma compagne, demain mon maître va répudier sa femme et me prendre pour femme. Elle lui demanda: Pourquoi ? (Elle répondit :) C'est parce qu'il a vu ses mains noircies. Elle lui dit: Hélas, idiote qui est dans le monde! Tes oreilles entendent-elles ce que dit ta bouche? Et si sa femme qui lui est chère, tu dis que parce qu'il voit ses mains noircies de manière temporaire, il veut la répudier, toi qui est noircie et noire depuis le sein de ta mère tous les

\footnotetext{
${ }^{69}$ Les parallèles renvoient à Ex 20, 3 .

${ }^{70}$ L'interprétation qui commence ici par «parce que » ne se rapporte pas aux versets précédemment cités (Qo 8,2; Ex 20, 2 et 7) et il s'agit plutôt d'une conclusion plus générale de l'homélie qui commence au début du paragraphe 6 . L'homélie porte sur le verset de Lv 25, 14 : « quand vous vendrez une vente (...) vous n'opprimerez (al tonu) pas chacun votre frère $»$. Le verbe makhar qui est habituellement lu au qal est compris, dans le début de l'homélie, comme étant au nif'al: «quand vous serez vendus... ». Le verset ferait allusion au fait que les Hébreux seront vendus comme esclaves aux Nations du monde. L'homélie commence donc par un passage négatif sur l'oppression de l'exil et se termine par un passage positif annonçant la vengeance eschatologique contre les ennemis d'Israël. Il est frappant de voir que le verset de Lv 25, 14 (al tonu, «vous n'opprimerez pas») comme celui d'Is 49, 26 (monaikh, "ceux qui te narguent») contiennent le même verbe YNH, au hif'il. Il est donc probable que les deux versets (celui du début et celui de la fin de l'homélie) sont liés : les Nations n'auraient pas dû opprimer/narguer Israël (Lv 25, 14), mais parce qu'elles l'ont fait, Dieu les châtiera durement dans les temps eschatologiques (Is 49, 26).

${ }^{71}$ Le dialogue entre la femme du Cantique des Cantiques et les filles de Jérusalem est compris comme un échange entre Israël et les Nations. Les filles de Jérusalem ont dû reprocher à la femme sa couleur noire. Elle rétorque que sa noirceur est accidentelle (due à l'effet du soleil), alors que celle de ses interlocutrices est permanente (comme chez les Africains). Il faut comprendre que les Nations du monde reprochent à Israël son idolâtrie (la faute du veau d'or). Israël répond que cette idolâtrie est un accident ponctuel, alors que l'idolâtrie fait corps avec l'identité des Nations.

${ }^{72}$ Une autre servante éthiopienne.
} 
jours de ta (vie), combien plus (il te répudierait). De même, les Nations du monde narguent Israël et disent: Cette nation a échangé sa gloire, ainsi qu'il est dit : «Et ils ont échangé leur gloire (pour l'image du bœuf mangeur d'herbe) » (Ps 106, 20). Israël leur répond : Et si nous sommes ainsi coupables de manière temporaire, combien plus en est-il pour vous. Israël dit encore aux Nations du monde: (Une parabole) est dite pour vous (à notre sujet). À quoi sommes-nous semblables ? À un fils de roi qui est sorti dans le désert de la ville. Le soleil a assommé sa tête et son visage est devenu livide. Il est entré (ensuite) dans la ville. En buvant un peu d'eau et en se baignant dans l'un des bains (de la ville), son corps a retrouvé sa blancheur et est revenu à sa beauté antérieure. De même, nous, c'est le soleil de l'idolâtrie qui nous a brunis, mais vous (les Nations), vous êtes brunes depuis le sein de votre mère. Même quand vous étiez dans le sein de votre mère, vous serviez les idoles. Comment? Au moment où la femme est enceinte, elle entre dans son temple idolâtre, elle s'incline et se prosterne devant l'idole, elle et son fils.

11. Shir ha-shirim Rabba, 8, 10, 2, ms Vatican, Biblioteca Apostolica ebr. 76

« Je suis une muraille » $(\mathrm{Ct} 8,10)$. Israël a dit devant le Saint, béni soit-il : Maître du monde, « Je suis une muraille », nous ferons se dresser les commandements et les bonnes actions comme une muraille. «Et mes seins sont comme des tours » $(\mathrm{Ct} 8,10)$, car dans le futur nous ferons se dresser maints groupes de justes semblables à nous dans ton monde. «Alors, je serai à ses yeux comme celle qui trouve la paix » $(\mathrm{Ct} 8,10)$. Pourquoi ? C'est parce que les Nations du monde narguaient Israël en disant: S'il en est ainsi ${ }^{73}$, pourquoi vous a-t-il exilé de sa terre? Pourquoi a-t-il détruit son Temple? Israël leur répondait (ainsi) : Nous sommes semblables à une fille de roi, qui est partie pour accomplir la « fête des anxieux » ${ }^{74}$ (regel redufim) dans la maison de son père (et) qui revient dans sa maison (de son époux) en paix.

\footnotetext{
${ }^{73}$ Il faut probablement comprendre : si vous avez tous ces mérites et que vous êtes le peuple élu, comme vous le prétendez.

${ }^{74} \mathrm{La}$ 《 fête des anxieux » est la première fête qui survient après le mariage (M. Jastrow, A Dictionary of the Targumim, the Talmud Babli and Yerushalmi, and the Midrashic Literature, New York, Judaica Press, 1992 [1903], p. 1453). Dans cette circonstance, la femme est désireuse (anxieuse) de retourner dans la maison de son père pour y célébrer la fête, mais ce n'est pas une répudiation, car elle revient ensuite dans la maison de son mari. De même, Israël peut être désireux de commettre des péchés et se retrouver en exil à cause de ses fautes, mais cela n'équivaut pas à un rejet complet de la part de Dieu. Il finira par retourner en paix dans la maison de son divin époux. Le texte fait peut-être
} 


\section{Midrash Hallel sur Ps $113,6^{75}$}

" (Dieu abaisse ses regards) sur le ciel et sur la terre ${ }^{76}$ (Ps 113, 6). (Le verset insiste sur la terre) parce que les Nations du monde ${ }^{77}$ narguent (les enfants d')Israël en leur disant : Il ne domine que les cieux ! Et (les enfants d')Israël leur répondent ainsi : Sortez et regardez le soleil qui est l'envoyé du Saint, béni soit-Il, il domine les montagnes et les collines. Si l'envoyé du Lieu est ainsi, le Saint, béni soit-Il, combien plus !

Si l'on excepte le premier et le dernier passage, tous ces textes sont tirés de Midrashim aggadiques, dont la rédaction finale est située en Palestine au $\mathrm{V}^{\mathrm{e}}$ ou au $\mathrm{VI}^{\mathrm{e}}$ siècle, c'est-à-dire à l'époque chrétienne byzantine $^{78}$. Quand ils ne sont pas anonymes, ils mettent plutôt en scène des amora'im palestiniens de la deuxième (250-290) et de la troisième générations (290-320). Ils ont tous en commun d'évoquer une attitude négative des Nations à l'égard d'Israël, exprimée par le verbe monin, qui est un participe masculin pluriel hif'il de la racine YNH. L'accompli correspondant, à la troisième personne du masculin singulier, est hona. Comme le montre la consultation des dictionnaires de référence (Larousse, Jastrow, Even-Shoshan), le verbe hona a une large palette de sens : tromper, abuser, exploiter, opprimer, tourmenter, irriter, vexer, narguer, se moquer. Dans les textes qui nous concernent, nous avons opté

allusion à la pratique romaine du regale repudium: voir $\mathrm{R}$. Kimelman, « Rabbi Yohanan and Origen... », op. cit., p. 590-591, n. 101.

${ }^{75}$ Bet ha-midrasch, éd. A. Jellinek, t. 5, Vienne, 1873, p. 92.

${ }^{76} \mathrm{Si}$ Dieu abaisse ses regards sur le ciel, il va de soi qu'il les abaisse aussi sur la terre, pourquoi le verset précise-t-il alors : « et sur la terre »?

${ }^{77}$ Le verset 4 du Psaume 113 mentionne les Nations : «L'Éternel est élevé au-dessus de toutes les Nations, sa gloire est dans le ciel ». Le couple des Nations et du ciel (verset 4) fait pendant à celui du ciel et de la terre (verset 6). Les Nations sont donc associées à la terre. En affirmant que Dieu ne règne pas sur la terre, les Nations refusent d'admettre qu'il règne sur elles.

${ }^{78}$ Le texte 1 provient du Talmud Yerushalmi, rédigé en 400 . Le texte 12 est tiré du Midrash Hallel. Ce Midrash est vraisemblablement un ouvrage médiéval, composé au $\mathrm{XII}^{\mathrm{e}}$ ou au XIII ${ }^{\mathrm{e}}$ siècle, même s'il donne l'impression d'avoir conservé des traditions anciennes, soit inédites, soit attestées ailleurs sous une autre forme (J. Costa, De l'importance des textes considérés comme mineurs: l'exemple du Midrash Hallel, Paris-Louvain, Peeters, 2013, p. 269-271 ; 309-310). M. Lerner envisage cependant la possibilité que le Midrash Hallel soit d'origine tannaïtique : "The Works of Aggadic Midrash and the Esther Midrashim », dans S. Safrai, Z. Safrai, J. Schwartz, P. J. Tomson (éd.), The Literature of the Sages. Second Part : Midrash and Targum. Liturgy, Poetry, Mysticism. Contracts, Inscriptions, Ancient Science and the Languages of Rabbinic Literature, Assen, Royal Van Gorcum-Fortress Press, 2006, p. 142. 
pour la traduction «narguer», qui, sans être idéale, a l'avantage de combiner à la fois le sentiment de supériorité et la moquerie.

Les textes réunis ici donnent donc un aperçu substantiel de l'antijudaïsme des Nations, tel qu'il était perçu par les rabbins antiques. L'attitude moqueuse et critique des Nations se traduit en effet par des déclarations, des questions ou des reproches embarrassants, auxquels les rabbins tentent de donner une réponse, souvent placée directement dans la bouche même d'Israël. Le contexte dans lequel apparaissent ces passages polémiques est toujours significatif. Pour le texte 1, il est question de Rabbi Yohanan qui fréquente les thermes, un lieu emblématique de la vie à la romaine. Tous les autres textes sont de nature midrashique. Le seul verset commenté qui emploie explicitement le verbe « narguer » est Is 49, 26 (t. 9) ${ }^{79}$. Pour les autres versets, le lien que le Midrash établit entre le ou les versets cités et la polémique entre les Nations et Israël est moins évident. Il est cependant clair que les versets mobilisés se réfèrent plus ou moins clairement aux non-juifs et aux Nations. Ps 111, 6 rappelle que Dieu a donné à son peuple l'héritage des Nations (t. 2). Les versets du texte 3 racontent comment Abraham, Jacob et David ont acheté des terrains à des Cananéens. L'insensé du Ps 39, 9 est bien sûr pour les rabbins le non-juif (t. 4). Les textes 4 et 5 partent de versets tirés du roman de Joseph, qui est le premier exemple connu de «juif de cour». Les textes 6, 10 et 11 commentent des versets du Cantique des Cantiques. Or, la lecture rabbinique de ce texte met habituellement l'accent sur l'amour exclusif que Dieu porte à Israël aux dépens des «rivales» que sont les autres Nations ${ }^{80}$. Si Dieu console Israël en Is 51, 12, c'est forcément des malheurs qu'il a subi de la part des Nations (t. 7). L'épisode du veau d'or, traité dans le texte 8 , est un exemple fameux d'idolâtrie juive, mais l'idolâtrie est la pratique qui définit les Nations en tant que telles. L'obéissance au roi divin, à laquelle fait allusion Qo 8, 2 (t. 9), peut rentrer en contradiction avec l'obéissance due aux rois des Nations ${ }^{81}$. Quant au Ps 113, 6, il contient dans son contexte immédiat une mention des Nations (Ps 113, 4). Quatre des

\footnotetext{
${ }^{79}$ Il est par ailleurs clair que le motif des Nations qui se moquent d'Israël est déjà biblique. Le verset de Ps 115, 2 et ses parallèles (Ps 42, 3 et $10 ; 79,10$; Jo 2, 17) ont probablement joué un rôle dans la conception de nos extraits, notamment les textes 7 et 12 .

${ }^{80}$ Voir J. Kaplan, My Perfect One. Typology and Early Rabbinic Interpretation of Songs of Songs, New York, Oxford University Press, 2015, p. 47-93.

${ }^{81}$ Voir le contexte immédiat du texte 9, qui évoque l'épisode des trois jeunes Hébreux et de Nabuchodonosor (Dn 3) et Ba-midbar Rabba, 14, 6; 15, 14.
} 
douze textes (t. 6-8, 10, 11) comportent aussi une ou des paraboles. Presque toutes sont de type royal (le roi étant l'équivalent de Dieu dans le récit) et parlent de mariage et de sexualité (c'est-à-dire de l'alliance entre Dieu et Israël). Deux paraboles parlent de calomnies mettant en question la vertu d'une ou plusieurs femmes (t. 6 et 8).

Dans huit des douze textes, la moquerie des Nations est considérée comme un fait, situé tantôt dans le passé («narguaient», t. 1, 6, 8, 11), tantôt dans le présent («narguent», t. 7, 9, 10, 12). Quand le passé est employé, le texte est parfois plus précis : les Nations du texte 1 sont témoins de l'errance des Hébreux dans le désert, celles du texte 8 sont identifiées aux mauvais "prosélytes qui sont montés avec Israël d'Égypte », celles du texte 6 sont vraisemblablement contemporaines de l'esclavage en Égypte. Seul le texte 9 situe la réponse à la moquerie des Nations dans le futur eschatologique ${ }^{82}$. Quatre textes (t. 2-5) se distinguent des autres par le fait qu'ils évoquent la moquerie des Nations comme quelque chose que l'on doit empêcher («afin qu'ils/elles ne narguent pas ») ou qui est considérée comme impossible («ne peuvent pas narguer »). Ces quatre textes sont tirés du même recueil, Be-reshit Rabba.

Le discours associé aux Nations se réduit généralement à une simple phrase. Dans le texte 1, il prend la forme de deux questions successives. Dans les textes 7 et 11, il consiste en une déclaration plus substantielle. Le texte 9 est le seul à ne pas faire parler les Nations, puisqu'il se contente de dire : «Dans le monde présent, les Nations du monde narguent Israël ». Dans presque tous les textes, l'objet du discours des Nations est Israël, qu'elles qualifient de voleur ou d'idolâtre et dont elles soulignent l'abaissement passé ou présent. Trois textes de Be-reshit Rabba définissent successivement Israël comme «une nation de voleurs » (t. 2), « une nation de gens abattus et repoussés », " une nation de lépreux » (t. 4) et « une nation de gens exilés et déplacés» (t. 5). L'accusation de vol concerne la prise de possession par les Hébreux de la terre de Canaan. Le reproche d'idolâtrie fait référence au péché originel d'Israël, la faute du veau d'or. Le texte 8 se contente de dire : «ils ont fait le veau d'or ». Le texte 10 fait usage d'une formule inspirée du Ps 106, 20 : «cette nation a échangé sa gloire ». La gloire qu'Israël a

\footnotetext{
${ }^{82}$ Deux textes situent la réponse dans le passé (t. 11) et le présent (t. 7), mais cette dernière comporte une composante eschatologique (t. 7 : la citation de Lv 24, 9 ; t. 11 : le retour en paix d'Israël dans la maison de son époux divin).
} 
échangée " pour l'image du bœuf » désigne soit son statut de peuple élu qu'il perd, soit le Dieu qu'il abandonne.

Le thème de l'abaissement d'Israël prend des formes variées. Dans le texte 4, Israël est à la fois abattu et repoussé (selon la lecture de Rabbi Hama be-Rabbi Hanina) et associé à la lèpre (selon la lecture de Rabbi Shemu'el bar Naḥman). Tout cela semble former un ensemble cohérent : des lépreux ne peuvent qu'être repoussés et abattus. Dans le texte 6, les Nations affirment que les Hébreux ont été contraints à des relations sexuelles forcées et qu'ils sont les « fils des Égyptiens ». Dans les textes 7 et 11 , les Nations rappellent à Israël les maux que Dieu a envoyés sur lui : oppression, mort, peine, mépris et souffrances (t. 7), exil et destruction du Temple (t. 11). Ce rappel vise cependant des objectifs différents : dans le texte 7, les Nations veulent intégrer les juifs en leur sein («Venez auprès de nous et nous ferons de vous des ducs, des gouverneurs et des commandants en chef»), dans le texte 11, elles polémiquent vraisemblablement contre leur statut de peuple élu, que contredit leur abaissement. Les textes 1 et 12 se distinguent des autres par le fait que le propos des Nations ne porte pas sur Israël mais sur Dieu. Dans le texte 1, les Nations s'interrogent : comment un objet aussi sacré que l'arche d'alliance peut-il cheminer à côté d'un cercueil ? Le texte 12 les voit au contraire être très affirmatives: Dieu «ne domine que les cieux ». Cette affirmation est probablement fondée sur l'abaissement d'Israël : si le Dieu des juifs avait réellement un pouvoir sur la terre, il n'aurait pas laissé son peuple se faire vaincre par les Nations. L'attitude des Nations à l'égard de Dieu varie d'un extrême à l'autre : respect de sa sainteté d'une part (t. 1), scepticisme et blasphème de l'autre (t. 12).

Au discours critique des Nations, les textes apportent des réponses. Dans quatre passages, c'est Israël qui répond directement aux Nations. Il souligne que le mérite lié à l'observance de la Tora prime sur les impératifs de la pureté rituelle (t. 1). Il distingue l'idolâtrie accidentelle du veau d'or de l'idolâtrie structurelle des Nations (t. 10). Il raconte une brève parabole qui montre que le peuple d'Israël n'a pas été définitivement abandonné par Dieu et qu'il est destiné à revenir dans la maison de son «époux » (t. 11). L'idolâtrie comme l'exil d'Israël sont temporaires et donc peu significatifs. Israël démontre la puissance de Dieu sur la terre, par un raisonnement a fortiori centré sur la figure du soleil (t. 12). Deux des versets commentés, tirés du Cantique des Cantiques, rapportent en fait, selon les rabbins, la réponse d'Israël aux Nations (t. $10:$ Ct 1, 6; t. $11:$ Ct 8, 10). Dans le texte 7, la réponse 
d'Israël aux Nations est indirecte : la lecture de la Tora le console et le conforte donc dans sa décision de rester fidèle à Dieu et de ne pas accepter les propositions tentantes des Nations. Les textes 6,8 et 9 attribuent la réponse à Dieu lui-même : il témoigne de la pureté sexuelle d'Israël (notamment par la proclamation de $\mathrm{Ct} 4,12$ ) ou il la prouve par l'action d'un ange (t. 6), il mène une enquête et parvient à innocenter Israël dans l'affaire du veau d'or (t. 8, comme le prouvent les versets cités), il punira les Nations pour leur attitude moqueuse à l'égard d'Israël (t. 9, comme l'affirme Is 49, 26). Le texte 2 est paradoxal. Alors qu'il affirme que le récit de la création a été révélé pour que les Nations ne narguent pas Israël, il mentionne ensuite une réponse d'Israël, ce qui suppose que les Nations l'ont nargué. Il est possible cependant que la réponse soit envisagée comme une simple possibilité : au cas où les Nations narguent quand même Israël, il pourrait répondre ainsi. Le texte comporte donc deux réponses : une réponse théologique attribuée à Dieu (fondée sur Gn 1, 1 et Ps 111, 6; le Dieu créateur est propriétaire du monde et donne la terre de Canaan à qui il veut) et une réponse historique attribuée à Israël (fondée sur Dt 2, 23 ; d'autres nations ont également pratiqué le vol de terres) ${ }^{83}$.

Le propre des textes 2 à 5 est qu'ils font de la réponse une arme dissuasive : elle empêche ou vise à empêcher la critique des Nations. Les textes 2 et 3 donnent ce pouvoir à des versets, les textes 4 et 5 à des actions de Joseph et de Dieu. Les textes 2 et 3 traitent du même problème : une accusation potentielle de vol. La solution du texte 2 , déjà vue, permet de régler le problème à la racine (le « vol» de la terre de Canaan est légitime), alors que celle du texte 3 est plus modeste (elle montre uniquement que trois lieux de la terre de Canaan n'ont pas été volés par les Hébreux). Les textes 4 et 5 abordent eux aussi une difficulté commune : le fait que les Hébreux sont une population repoussée ou déplacée. Ils la résolvent en revanche par la même méthode : Joseph dans un cas et Dieu dans l'autre suscitent des mouvements de population chez les Nations, de manière à rapprocher leur condition de celle d'Israël et à empêcher ainsi toute critique de leur part. Une telle stratégie suppose que Joseph savait à l'avance que l'avenir d'Israël comporterait une longue période d'exil. Le texte 4 suggère que c'est Israël lui-même qui a

\footnotetext{
${ }^{83}$ Voir M. Kister, «The Fate of the Canaanites and the Despoliation of the Egyptians. Polemics among Jews, Pagans, Christians, and Gnostics : Motifs and Motives ", dans K. Berthelot, J. E. David, M. Hirshman (éd.), The Gift of the Land and the Fate of the Canaanites in Jewish Thought, Oxford-New York, Oxford University Press, 2014, p. 74.
} 
demandé à Dieu de frapper les Nations, soit dans le présent (au moment où il est lui-même frappé), soit dans le passé (ce qui implique, là aussi, un savoir prophétique).

Qui sont les gens des Nations mis en scène dans les douze textes? Certains passages (t. 1, 6, 8) les identifient plus ou moins explicitement à des contemporains de l'époque de l'exode ou du désert. Les autres sont beaucoup plus elliptiques. Il est également frappant que les difficultés soulevées par les Nations le sont aussi par les rabbins eux-mêmes dans leurs ouvrages aggadiques, dans un contexte non polémique, du moins en apparence. Le texte 1 traite de l'observance d'un commandement juif (celui des tefillin) et aborde une question typiquement rabbinique, celle des tensions qui existent entre deux types de pureté, la pureté rituelle et le mérite qui découle de l'observance plus générale de la Tora ${ }^{84}$. Dans le même texte, les Nations n'hésitent pas à reprendre les termes mêmes qu'emploient Israël pour qualifier Dieu et Joseph : « Est-il possible au coffre du mort de se déplacer avec le coffre du Vivant des mondes? ». Dans les textes 7 et 11, les affirmations des Nations (t. $7:$ « combien de peine a-t-il fait venir sur vous ? Combien de mépris a-t-il fait venir sur vous? Combien de souffrances a-t-il fait venir sur vous?»; t. 11: «pourquoi vous a-t-il exilé de sa terre? Pourquoi a-t-il détruit son Temple ? ») font penser à une idée massivement présente dans la Bible et chez les rabbins : les victoires des Nations ne s'expliquent pas par leur puissance, mais par le fait que Dieu, en se servant d'elles, punit Israël pour ses fautes ${ }^{85}$.

Des événements comme la faute du veau d'or, la destruction du Temple ou encore l'exil et ses souffrances ont suscité de nombreux commentaires visant à les expliquer, de manière à préserver le statut

\footnotetext{
${ }^{84}$ Voir par exemple A. Tropper, Like Clay in the Hands of the Potter. Sage Stories in Rabbinic Literature (hébreu), Jerusalem, The Zalman Shazar Center for Jewish History, 2011, p. 27-45.

${ }^{85}$ Voir S. J. D. Cohen, "The Destruction: From Scripture to Midrash », dans The Significance of Yavneh and Other Essays in Jewish Hellenism, Tübingen, Mohr Siebeck, 2010, p. 22-43; C. Touati, «Les deux théories de Maïmonide sur la providence », dans Prophètes, talmudistes, philosophes, Paris, Le Cerf, 1990, p. 190191. La question initiale ( "Jusqu'à quand serez-vous opprimés par votre Dieu... ? ») et la suite du texte 7 suggèrent cependant que les souffrances subies par Israël ne sont pas des punitions, bien au contraire : c'est parce qu'Israël reste fidèle à la loi divine qu'il souffre dans les mains des Nations. Ces souffrances viennent donc de Dieu uniquement de manière indirecte. Si les Nations du texte se confondent avec les païens, il est possible qu'il y ait aussi une moquerie à la manière de Ps 115, 2 : où est ce Dieu impuissant qui vous laisse souffrir sans intervenir?
} 
d'Israël comme le peuple central de l'histoire, élu par Dieu et voué à être sauvé à la fin des temps ${ }^{86}$. Le texte 8 critique les prosélytes, comme d'autres passages de la littérature rabbinique ${ }^{87}$. Ceci étant dit, il est inévitable de se demander si le discours anti-juif des Nations, qui se déploie dans nos textes, n'est pas surtout le reflet de ce que disaient deux grands adversaires des rabbins: les païens d'une part et les chrétiens d'autre part, et ce même quand le passage situe la scène dans le passé ou présente la critique des Nations comme une simple possibilité qu'il faut empêcher.

L'accusation d'avoir volé la terre de Canaan (t. 2, 3) est à première vue un motif d'origine païenne, qui remonte au moins au $\mathrm{II}^{\mathrm{e}}$ siècle avant notre ère, comme le suggère un ensemble de sources réuni et étudié par M. Kister (les Jubilés, Philon, Flavius-Josèphe, Procope) ${ }^{88}$. Il est même possible que certains païens se soient fondés sur la Bible pour appuyer leurs revendications, puisqu'elle reconnaît que les Cananéens sont les premiers habitants de la terre d'Israël et parle des biens que les Hébreux ont emportés d'Égypte au moment de l'Exode ${ }^{89}$. L'idée que les juifs sont des lépreux et «des gens (...) repoussés », exprimée dans le texte 4, est également de provenance païenne. C'est en effet de cette manière que certains auteurs païens comprennent l'Exode: les juifs auraient été expulsés d'Égypte à cause de leur lèpre. D'autres passages de la littérature rabbinique confirment que les rabbins connaissent bien cette autre version de l'Exode et qu'ils s'efforcent de lui apporter une

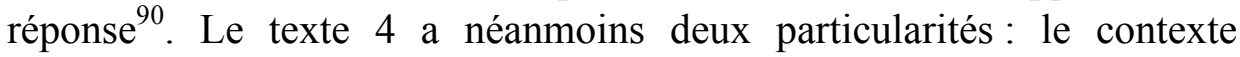
égyptien n'est qu'indirectement présent et les rabbins admettent la

\footnotetext{
${ }^{86}$ Sur tous ces thèmes, voir E. E. Urbach, Les Sages d'Israël. Conceptions et croyances des maitres du Talmud, Paris-Lagrasse, Le Cerf, 1996, p. 542-581. Concernant la terre de Canaan, le Sifra (Mekhilta de-'arayyot, Qedoshim, sur Lv 20, 24, éd. Weiss, p. 93c) précise qu'elle appartenait originellement au lot de Sem et que les Cananéens n'en étaient que les gardiens jusqu'à l'arrivée des Hébreux.

${ }^{87}$ Voir G. G. Porton, The Stranger within Your Gates. Converts and Conversion in Rabbinic Literature, Chicago, University of Chicago Press, 1994.

${ }^{88}$ M. Kister, «The Fate of the Canaanites... », op. cit., p. 69-74, 77-78.

${ }^{89}$ M. Kister, «The Fate of the Canaanites... », op. cit., p. 90.

${ }^{90}$ M. Kister, «The Fate of the Canaanites... », op. cit., p. 77, p. 100, n. 85. Le texte 4 affirme pourtant que l'action divine a pour but d'éviter l'accusation païenne, alors que cette accusation est bien réelle et que les rabbins le savent. Il est également possible que les rabbins connaissent une autre histoire polémique, prisée par les Grecs, celle de Moïse et de l'âne: voir J. Costa, "Canon et traduction (Septante, Aquila): des traditions rabbiniques en rapport avec le judaïsme synagogal ? », dans C. Clivaz, S. C. Mimouni, B. Pouderon (éd.), Les judaïsmes dans tous leurs états aux Ier-IIIème siècles (les Judéens des synagogues, les chrétiens et les rabbins). Actes du colloque de Lausanne, 12-14 décembre 2012, Turnhout, Brepols, 2015, p. 393.
} 
présence de lépreux au sein d'Israël. Le terme meșora im, que nous avons traduit par «lépreux », est en fait plus ambigu. Le meṣora ' est un homme juif, atteint d'une maladie de peau appelée șara 'at, qui le rend impur rituellement. Il est par ailleurs évident que la șara 'at est une maladie de peau qui n'a rien à voir avec la lèpre ${ }^{91}$. Il est donc possible que dans notre texte le terme meșora 'soit employé dans son sens usuel, ce qui est aussi une manière de répondre aux Nations : Israël n'est pas un peuple de lépreux, mais il est susceptible d'être affecté par une autre maladie de peau qui le rend impur, alors qu'elle n'a pas cet effet sur les gens des Nations.

Cette lecture est confirmée par le fait que le texte emploie le terme de meșora' pour Israël, alors qu'il fait usage d'un autre terme pour les Nations : «galeux ». Elle n'est pas cependant certaine, car il arrive aussi aux rabbins d'associer la șara'at et la vraie lèpre ${ }^{92}$. Comme le texte 5 a un contexte égyptien explicite et qu'il définit Israël comme un peuple de gens « exilés et déplacés ", il est tentant de le rapprocher du texte 4 et d'y voir une autre allusion aux contre-histoires païennes de l'Exode. Le texte 6, où les Nations identifient Israël aux «fils des Égyptiens », n'a pas vraiment de parallèle chez les païens et les chrétiens, au point qu'on pourrait y voir un exemple de transfert sur les Nations d'une préoccupation qui est en fait interne au monde rabbinique (la pureté sexuelle d'Israël). Il est possible néanmoins que, comme les deux passages précédents, il se fasse l'écho d'une réécriture païenne de 1'Exode qui n'aurait pas été préservée par ailleurs ${ }^{93}$.

Contrairement aux textes 2-6, que nous avons rapprochés de la judéophobie païenne, les textes 7-11 semblent se référer à l'antijudaïsme chrétien, même si, dans le détail, les choses sont parfois un peu plus compliquées. Dans le texte 7, la mention de Rabbi Yoḥanan (250-290), de l'expression «votre Dieu» (les chrétiens ont a priori le même Dieu que les juifs) et de l'accès à des fonctions publiques (ducs, gouverneurs, commandants en chef) font plutôt penser au contexte de la Palestine

\footnotetext{
${ }^{91}$ Sur tous ces points, voir J.-P. Messali, La «lèpre» dans les écrits bibliques et rabbiniques. Aspects historiques, textuels et rituels, Doctorat de l'Université de Paris 3 , soutenu le 13 avril 2016, p. 66.

${ }_{92}$ Voir J.-P. Messali, La « lèpre »..., op. cit., p. 338-341.

${ }^{93}$ Pour cette dernière hypothèse, voir M. Kister, «The Fate of the Canaanites... », op. cit., p. 100, n. 84. Kister remarque également que dans la réécriture de l'Exode par Manéthon, les Hébreux sont aussi les « fils des Égyptiens », mais pas pour les mêmes raisons que dans le texte 6, car les Hébreux de 1'Exode sont tout simplement remplacés par des lépreux égyptiens qui sont expulsés de leur propre pays.
} 
placée sous hégémonie païenne ${ }^{94}$. L'hypothèse chrétienne est cependant envisageable, si l'on tient plutôt compte de la date de rédaction du recueil contenant le texte 7 (la Pesiqta de-rab Kahana) : le texte ferait alors allusion au fait que les juifs ont été exclus de certaines fonctions publiques dans l'Empire romain christianisé ${ }^{95}$. Le fait qu'Israël trouve la consolation dans les synagogues fait penser à la période byzantine en Palestine, marquée par une nette croissance de la vie synagogale ${ }^{96}$. Le motif des souffrances que Dieu fait venir sur les juifs peut être aussi compris dans une perspective chrétienne : Dieu châtie leur obstination à pratiquer une religion et une loi dépassées et à ne pas reconnaître la vérité du christianisme ${ }^{97}$. Dans le texte 8 , les Nations reprochent à Israël la faute du veau d'or, ce qui est une attitude fréquente chez les Pères de l'Église ${ }^{98}$. Les «prosélytes », sortis d'Égypte avec Israël, peuvent facilement représenter les tenants d'une religion au prosélytisme ardent. Le texte 10, qui commente $\mathrm{Ct} 1,6$, voit Israël opposer son idolâtrie accidentelle à l'idolâtrie constante des Nations. Cet argument paraît à première vue s'appliquer plutôt aux païens qu'aux chrétiens. L'accusation d'idolâtrie est néanmoins hautement polémique et des «monothéistes» n'hésitent pas à la lancer contre d'autres « monothéistes ${ }^{99}$.

Elle peut aussi faire allusion à l'origine païenne d'un grand nombre de chrétiens ou à des pratiques idolâtres effectives dans le cadre de

\footnotetext{
${ }^{94}$ Voir I. Baer, «'Am yisra'el, ha kenesiyya ha-noșrit we-ha-qesarut ha-romit mi-yme Septimius Severus we-'ad "pequddat ha-sablanut" shel shenat 313 », Zion 21, 1956, p. 19, 30. Baer se réfère à un passage du Contre Celse, 8, 73, où Celse préconise l'extermination des chrétiens qui menacent la survie de l'Empire, sauf s'ils acceptent de le servir, notamment dans l'armée. Celse se tourne vers les chrétiens et leur dit: Venez nous rejoindre ! Selon Baer, les juifs se sont retrouvés dans une situation semblable à l'époque de Rabbi Yoḥanan, comme le montre le texte 7 (Rabbi Yoḥanan est un contemporain de la persécution de Dèce et de la guerre entre Rome et la Perse). Baer affirme néanmoins aussi que «Rabbi Yoḥanan a déjà répondu à l'argument de la répudiation (d'Israël défendu par Origène) » dans le texte 7 (p. 19).

${ }^{95}$ Voir C. Nemo-Pekelman, «Le législateur chrétien a-t-il persécuté les juifs ? », dans M.-F. Baslez, Chrétiens persécuteurs. Destructions, exclusions, violences religieuses au $I V^{e}$ siècle, Paris, Albin Michel, 2014, p. 158-160.

${ }^{96}$ Voir L. I. Levine, The Ancient Synagogue. The First Thousand Years, New HavenLondres, Yale University Press, 2000, p. 194-231.

${ }^{97}$ Le contenu du discours des Nations, dans le parallèle de Ekha Rabba, a une tonalité encore plus chrétienne.

${ }^{98}$ Voir L. Smolar, M. Aberbach, « The Golden Calf Episode in Postbiblical Literature », Hebrew Union College Annual 39, 1968, p. 96-101.

${ }^{99}$ Voir G. R. Hawting, The Idea of Idolatry and the Emergence of Islam. From Polemic to History, Cambridge, Cambridge University Press, 1999, p. 67-87.
} 
persécutions romaines ${ }^{100}$. Les versets de Ct 1, 5- 6 ont suscité l'intérêt d'Origène, qui identifie la femme « noiraude » à l'Église et les « filles de Jérusalem » aux juifs. Origène reconnaît que les chrétiens n'ont pas une ascendance prestigieuse, mais la femme noiraude est cependant belle par sa foi et son repentir et elle est finalement préférée par l'amant divin ${ }^{101}$. Le sens du passage du Cantique des Cantiques faisait donc l'objet de débats entre les rabbins et les Pères. Selon le texte 11, les Nations voient probablement dans l'exil d'Israël et la destruction du Temple le signe qu'Israël n'est plus le peuple élu de Dieu. Tout cela reflète bien les thèmes courants de l'antijudaïsme chrétien. La réponse des rabbins va aussi dans ce sens : Israël est une épouse qui est éloignée temporairement de son mari et non une femme répudiée. Or, Origène a défendu la thèse inverse selon laquelle Dieu a écarté définitivement son épouse juive, ce que montrent à la fois la destruction du Temple et l'exil ${ }^{102}$. Le texte 12 est ambigu. Il peut faire allusion aux chrétiens gnostiques, selon lesquels la divinité la plus élevée ne gouverne pas la terre. Ce texte 12 identifie cependant ce dieu céleste au Dieu d'Israël, alors que les gnostiques croient exactement l'inverse : le démiurge, qui domine la terre, est le Dieu des juifs ${ }^{103}$. Construites largement par les rabbins, les Nations du passage seraient plutôt identifiées à une puissance païenne, qui, à cause des victoires qu'elle a remportées sur Israël, estime, dans une logique hénothéiste, que le dieu des juifs, à l'inverse du sien, est impuissant sur la terre.

En conclusion, les douze textes présentés ici contiennent bel et bien ce que les linguistes appellent un «contre-discours rapporté direct», souvent suivi de sa riposte. M. Azaiez a récemment montré l'importance

\footnotetext{
100 Sur les deux dernières explications, voir R. Kimelman, « Rabbi Yohanan and Origen... », op. cit., p. 594 et n. 112 ; I. Baer, "'Am yisra'el, ha kenesiyya hanoṣrit... ", op. cit., p. 19 (Baer évoque les persécutions de Dèce et de Maximin, où même les enfants ont dû sacrifier aux dieux).

${ }^{101}$ Voir à la fois les Homélies et le Commentaire d'Origène sur Cantique des Cantiques, 1, 5-6 ; E. Urbach, « The Homiletical Interpretations of the Sages and the Expositions of Origen on Canticles and the Jewish-Christian Disputation ", dans Collected Writings in Jewish Studies, Jérusalem, Varda Books, 2009, p. 334-336 ; R. Kimelman, « Rabbi Yohanan and Origen... », op. cit., p. 593.

102 Voir Origène, Homélies sur Jérémie, 4, 2 ; R. Kimelman, « Rabbi Yohananan and Origen... », op. cit., p. 589.

103 Voir par exemple T. Rasimus, «The Archangel Michael in Ophite Creation Mythology », dans L. Jenott, S. K. Gribetz (éd.), Jewish and Christian Cosmogony in Late Antiquity, Tübingen, Mohr Siebeck, 2013, p. 107-125.
} 
de ce contre-discours à l'intérieur du Coran ${ }^{104}$. Comme dans le cas du Coran, l'adversaire n'est pas clairement identifié. Selon M. Azaiez, « ne pas désigner l'adversaire, c'est construire l'identité de celui-ci à partir des énoncés modalisés du texte. Autrement dit, ne pas nommer l'adversaire, c'est lui affecter une identité construite par les qualificatifs que lui donne le Coran ${ }^{105}$. Dans nos douze textes, la figure des Nations est en effet en partie une construction rabbinique. Si l'on excepte cependant certains passages (essentiellement les textes 1 et 12), le gros de ces textes reflète aussi un discours anti-juif réel, assez bien attesté chez les païens ou chez les chrétiens. Il est frappant que le Midrash le plus ancien de notre échantillon, Be-reshit Rabba, se concentre clairement sur la judéophobie païenne, alors que les autres Midrashim, légèrement plus tardifs, mettent plutôt l'accent sur l'antijudaïsme chrétien $^{106}$. Que le contre-discours rapporté direct soit passé ou présent, les rabbins semblent viser à chaque fois des interlocuteurs contemporains et les textes étudiés ici sont finalement l'écho assez fidèle des polémiques qui ont opposé les principaux groupes religieux dans la période de l'Antiquité tardive.

\footnotetext{
${ }^{104}$ M. Azaiez, Le contre-discours coranique, Berlin-Boston, De Gruyter, 2015.

${ }^{105}$ M. Azaiez, Le contre-discours ..., op. cit., p. 293.

${ }^{106}$ Voir M. Kister, «The Fate of the Canaanites... », op. cit., p. 100, n. 84.
} 